\title{
Action growth of dyonic black holes and electromagnetic duality
}

\author{
Hai-Shan $\operatorname{Liu}^{a, b}$ and H. Lü ${ }^{a}$ \\ ${ }^{a}$ Center for Joint Quantum Studies and Department of Physics, School of Science, \\ Tianjin University, Tianjin 300350, China \\ ${ }^{b}$ Institute for Advanced Physics $\&$ Mathematics, Zhejiang University of Technology, \\ Hangzhou 310023, China \\ E-mail: hsliu.zju@gmail.com, mrhonglu@gmail.com
}

ABSTRACT: Electromagnetic duality of Maxwell theory is a symmetry of equations but not of the action. The usual application of the "complexity = action" conjecture would thus lose this duality. It was recently proposed in arXivid:1901.00014 that the duality can be restored by adding some appropriate boundary term, at the price of introducing the mixed boundary condition in the variation principle. We present universal such a term in both first-order and second-order formalism for a general theory of a minimally-coupled Maxwell field. The first-order formalism has the advantage that the variation principle involves only the Dirichlet boundary condition. Including this term, we compute the onshell actions in the Wheeler-De Witt patch and find that the duality is preserved in these actions for a variety of theories, including Einstein-Maxwell, Einstein-Maxwell-Dilaton, Einstein-Born-Infeld and Einstein-Horndeski-Maxwell theories.

KEYwords: Black Holes, AdS-CFT Correspondence, Gauge-gravity correspondence, Classical Theories of Gravity

ARXIV EPRINT: 1905.06409 


\section{Contents}

1 Introduction 1

2 First-order and second-order formalisms 3

3 Einstein-Maxwell thoery $\quad 6$

4 Einstein-Maxwell-Dilaton theory $\quad 8$

4.1 Dyonic black hole with $a=1 \quad 9$

4.2 Dyonic black hole with $a=\sqrt{3} \quad 9$

5 Einstein-Born-Infeld theory 10

6 Einstein-Maxwell-Horndeski theory $\quad 13$

$\begin{array}{lll}\text { 6.1 Charged AdS planar black hole } & 13\end{array}$

$\begin{array}{ll}6.2 \text { Charged AdS spherical black hole } & 14\end{array}$

7 Conclusions $\quad 16$

\section{Introduction}

The AdS/CFT correspondence builds a bridge between classical gravity in asymptotic anti-de Sitter (AdS) spacetime and some strongly-coupled conformal field theory (CFT) living on its boundary [1-4]. It provides a new tool for studying the dynamics of various strongly coupled quantum field theories [5-8]. Recently, holographic connections between the quantum complexity of a state on the boundary and some physical quantities in the bulk theory have attracted considerable attention. Conjectures such as "complexity = volume" (CV) $[9,10]$ and "complexity = action" (CA) [11, 12] have been proposed. Many works have been done to widely explore the properties of the holographic complexity related to these two conjectures [13-36] and further to generalize these two conjectures [37-42].

The CA conjecture states that the complexity of the boundary state is related to the on-shell classical gravitational action evaluated on the so-called "Wheeler-DeWitt (WDW) patch" of the asymptotically-AdS black hole, namely

$$
\mathcal{C}=\frac{I_{\mathrm{WDW}}}{\pi} .
$$

The WDW patch is a spacetime area enclosed by light rays, as illustrated in figure 1 .

It was well known that in gravities or supergravities, many global symmetries can be realized only at the level of equations, but not directly in the action. Indeed the well-known Cremmer-Julia $E_{7(+7)}$ global symmetry of maximum supergravity in four dimensions is a 


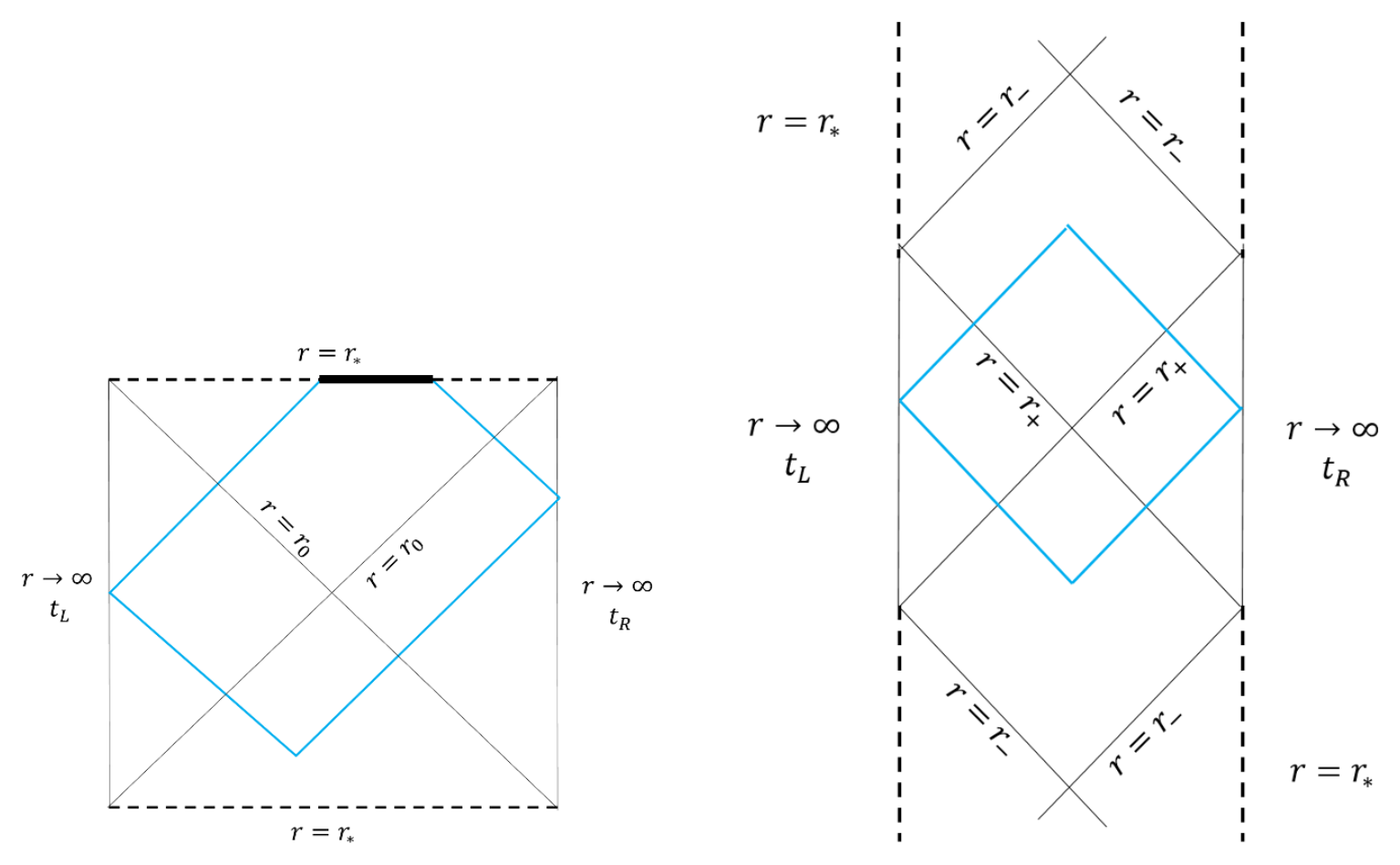

Figure 1. Plots of Wheeler-DeWitt patches. The left panel shows the causal structure of Schwardzchild-like black hole which has only one event horizon located at $r_{0}$, the region enclosed by blue lines is the WDW patch. The right panel shows the causal structure of Reissner-Nordstrorm like black hole which has two horizons located at $r_{+}$and $r_{-}$, and the WDW patch is the area enclosed by the blue lines.

symmetry of equations. The simplest example to illustrate this is perhaps the Maxwell theory in general curved spacetime with the Lorentzian signature. The action is

$$
I=\frac{\kappa}{16 \pi} \int_{M} d^{4} x \sqrt{-g}\left(-\frac{1}{4} F^{2}\right), \quad F=d A
$$

The equation of motion and Bianchi identity, i.e.

$$
d * F=0, \quad d F=0
$$

can be interchanged by interchanging $F$ and $* F$, giving rise to the electromagnetic duality. However, this symmetry cannot be realized in the action for two reasons. The first is that the fundamental field in Lagrangian is the gauge potential $A$ rather than $F$; the second is that in Lorentzian signature, we have the identity

$$
(* F)^{2}=-F^{2} .
$$

Indeed the electric and magnetic charges $\left(Q_{e}, Q_{m}\right)$ contribute symmetrically to the energymomentum tensor, e.g. $Q_{e}^{2}+Q_{m}^{2}$, but they contribute anti-symmetrically to the on-shell action, e.g. $Q_{e}^{2}-Q_{m}^{2}$. It follows that the standard application of the CA conjecture would loose the duality in the Einstein-Maxwell theory. This disappearance of the electromagnetic 
duality in holographic complexity from the CA conjecture was recently pointed out in [43] and [44].

There is more than one way to restore the electromagnetic duality, see e.g. [45-47]. A double-field formalism can make the duality manifest even in the off-shell action at the price of introducing extra degrees of freedom that need to be removed by constraints [48]. For the on-shell action, the restoration of the duality is simpler and can be done simply by introducing a Maxwell boundary term [44]

$$
\frac{\kappa \gamma}{16 \pi} \int_{\partial M} d \Sigma_{\mu} F^{\mu \nu} A_{\nu}
$$

with appropriate coefficient $\gamma$. Unlike the Gibbons-Hawking surface term [49], this boundary term was not required by the variation principle. In fact except for $\gamma=0,1$, the variation principle would have to deal with mixed Dirichlet and Neumann boundary conditions, which typically requires extra degrees of freedom on the boundary. The $\gamma=1$ case was introduced in [49-52] and it has an effect of performing the Legendre transformation in black hole thermodynamics associated with the electric charge $Q_{e}$ and its canonical potential $\Phi_{e}$.

It was demonstrated for the RN-AdS black hole [44] that the on-shell action can recover the full electromagnetic duality for appropriate $\gamma$. This leads to an important question whether there is a universal $\gamma$ for restoring the electromagnetic duality in the on-shell action. The situation for purely electric or purely magnetic AdS black holes in a family of Einstein-Maxwell-Dilaton (EMD) theory was also examined in [44].

In this paper, we examine a variety of theories that exhibit the electromagnetic duality at the level of equations of motion. These theories all admit dyonic AdS black holes, which allow us to evaluate the on-shell action and determine whether there is a universal term that can restore the duality in the on-shell action.

We first propose in section 2 a first-order formalism for a general theory of the Maxwell field that couples to gravity minimally. In this first-order system, we can add a total derivative term to restore the electromagnetic duality of the on-shell action. The advantage of the first-order formalism is that the variation principle involves only the Dirichlet boundary condition. We show that the on-shell action is precisely equivalent to the one with the Maxwell boundary term in the second-order formalism. This yields a general formula for the required boundary term for restoring the electromagnetic duality. Since we are unable to give a mathematical proof for this formula, in subsequent sections, we examine the on-shell action for dyonic black holes in Einstein-Maxwell, Einstein-Maxwell-Dilaton, Einstein-Born-Infeld and Einstein-Hordenski-Maxwell theories and confirm our conjecture. In doing so, we obtain the explicit late-time action growth rate of the dyonic black holes in these theories. We conclude the paper in section 7 .

\section{First-order and second-order formalisms}

In this section, we set up the general formalism for calculating the on-shell action involving the Maxwell field. We shall apply this formalism throughout this paper to evaluate the 
on-shell actions for various black hole solutions. We consider a general theory of the minimally-coupled Maxwell field in four-dimensional curved spacetime, We begin with the first-order formalism and the action is given by

$$
\mathcal{I}_{F}=\int_{M} d^{4} x \sqrt{-g}\left[\mathcal{L}_{F}\left(F_{\mu \nu}\right)-\frac{1}{2} \epsilon^{\mu \nu \rho \sigma} \nabla_{\mu}\left(F_{\nu \rho}\right) B_{\sigma}+\frac{1}{2} \gamma \epsilon^{\mu \nu \rho \sigma} \nabla_{\mu}\left(F_{\nu \rho} B_{\sigma}\right)\right] .
$$

Here the antisymmetric Maxwell field strength $F_{\mu \nu}$ is a fundamental field and $B_{\mu}$ is an auxiliary field. The $\gamma$-term is a total derivative of a bilinear term and gives no contribution to the equations of motion; however, it can alter the on-shell action. Variation of the action with respect to $B_{\mu}$ gives the Bianchi identity

$$
\nabla_{[\mu} F_{\nu \rho]}=0
$$

which implies that $F=d A$. Variation of $F_{\mu \nu}$ gives

$$
\widetilde{F}^{\mu \nu}=\epsilon^{\mu \nu \rho \sigma} \nabla_{\rho} B_{\sigma}, \quad \text { with } \quad \widetilde{F}^{\mu \nu} \equiv-2 \frac{\partial \mathcal{L}_{F}}{\partial F_{\mu \nu}} .
$$

Consequently, $\widetilde{F}^{\mu \nu}$ satisfies the equation of motion

$$
\nabla_{\mu} \widetilde{F}^{\mu \nu}=0
$$

Applying the Bianchi identity and equation of motion, the on-shell action is

$$
\begin{aligned}
\mathcal{I}_{\text {on-shell }}^{F} & =\int_{M} d^{4} x \sqrt{-g}\left[\mathcal{L}_{F}+\frac{1}{2} \gamma \epsilon^{\mu \nu \rho \sigma} F_{\mu \nu} \nabla_{\rho} B_{\sigma}\right] \\
& =\int_{M} d^{4} x \sqrt{-g}\left[\mathcal{L}_{F}+\frac{1}{2} \gamma \widetilde{F}^{\mu \nu} F_{\mu \nu}\right] .
\end{aligned}
$$

It should be emphasized that in this first-order formalism, the standard Dirichlet boundary condition should be adopted in the application of the variation principle, namely the variation of the fundamental fields $\delta F_{\mu \nu}$ and $\delta B_{\mu}$ both vanish on the boundary.

Alternatively, we consider the second formalism where $F=d A$ and $A$ is the Maxwell potential. In this case, the Bianchi identity is automatic. The action is

$$
\mathcal{I}_{A}=\int_{M} d^{4} x \sqrt{-g} \mathcal{L}_{F}\left(F_{\mu \nu}\right)+\int_{\partial M} d \Sigma_{\mu} S^{\mu},
$$

where $d \Sigma_{\mu}$ is the infinitesimal boundary volume element pointing to the normal direction of the boundary, and $S^{\mu}(A)$ is some appropriate boundary term that is to be determined. The variation with respect to $A_{\nu}$ gives (after integrating out the total derivative)

$$
\delta \mathcal{I}_{A}=\int_{M}\left(\nabla_{\mu} \widetilde{F}^{\mu \nu}\right) \delta A_{\nu}+\int_{\partial M} d \Sigma_{\mu}\left(-\widetilde{F}^{\mu \nu} \delta A_{\nu}+\delta S^{\mu}\right)
$$

Thus the equation of motion is (2.4), the same as in the first-order formalism. Furthermore, the boundary structure of $\delta \mathcal{I}_{A}$ suggests that we can take

$$
S^{\mu}=\gamma \widetilde{F}^{\mu \nu} A_{\nu}
$$


In particular, in Maxwell theory with $\widetilde{F}_{\mu \nu}=F_{\mu \nu}$, setting $\gamma=1$ gives the Legendre transformation interchanging the role of $\widetilde{F}^{\mu \nu}$ and $A_{\nu}$. The Dirichlet boundary condition $\left.\delta A_{\mu}\right|_{\partial M}=$ 0 is then switched to become the Neumann boundary condition $\left.n^{\mu} \partial_{\mu} \delta A_{\nu}\right|_{\partial M}=0$. In the first law of black hole thermodynamics, it becomes the Legendre transformation interchanging the role of the electric charge and its chemical potential. Here we let the constant $\gamma$ be arbitrary.

The on-shell action is then

$$
\begin{aligned}
\mathcal{I}_{A} & =\int_{M} d^{4} x \sqrt{-g} \mathcal{L}_{F}+\gamma \int_{\partial M} d \Sigma_{\mu} \widetilde{F}^{\mu \nu} A_{\nu} \\
& =\int_{M} d^{4} x \sqrt{-g}\left(\mathcal{L}_{F}+\gamma \nabla_{\mu}\left(\widetilde{F}^{\mu \nu} A_{\nu}\right)\right) .
\end{aligned}
$$

The Stock's theorem was applied in the second equality. It is clear that the on-shell actions (2.5) and (2.9) are the same. The price to pay in the second-order formalism is that one has to adopt the mixed Dirichlet and Neumann boundary condition, which typically requires additional degrees of freedom on the boundary. However, in terms of the evaluation of the on-shell action, the two formalisms give the identical result and it is more straightforward to use (2.9) to evaluate the action.

Thus we see that the action of the (general) Maxwell theory is not uniquely defined. In the first-order formalism, the ambiguity amounts to a total derivative, with an arbitrary dimensionless constant $\gamma$. In the second-order formalism, it represents itself as a surface term. However, this surface term is not required by the variation principle such as the Gibbons-Hawking term that must have a specific coefficient. The effect is like a Legendre transformation in black hole thermodynamics at the price of introducing mixed boundary conditions. The two different approaches lead to the same on-shell action with an additional $\gamma$ term.

The purpose of this paper is to study the effect of this $\gamma$-term on the on-shell action. For simple theory such as the Maxwell theory with $\mathcal{L}_{F}=-\frac{1}{4} F^{2}$, we have $\tilde{F}_{\mu \nu}=F_{\mu \nu}$ and hence

$$
\mathcal{I}_{\text {on-shell }}^{F}=\int_{M} d^{4} x \sqrt{-g}\left(-\frac{1}{4}(1-2 \gamma) F^{2}\right)
$$

which vanishes when $\gamma=\frac{1}{2}$. Since the electric and magnetic contributions to the energymomentum tensor and hence the metric are symmetric, it follows that the on-shell action with $\gamma=\frac{1}{2}$ is symmetric with respect to the electric and magnetic charges. However, for general $\mathcal{L}_{F}$, the quantity $\mathcal{I}_{\text {on-shell }}^{F}$ does not necessary vanish even when $\gamma=\frac{1}{2}$. This leads to a question whether it still has the electromagnetic duality. We shall provide sufficiently enough examples that support the conjecture that $\gamma=\frac{1}{2}$ is the universal coefficient for restoring the electromagnetic duality for the on-shell action, provided that the electromagnetic duality does exist in the equations of the theory.

In four dimensions, there are more total derivatives that one can consider. One is the $F \wedge F$ term and the other is the Gauss-Bonnet combination

$$
\begin{aligned}
I_{F \wedge F} & =\frac{\kappa}{16 \pi} \tilde{\gamma} \int_{M} d^{4} x \frac{1}{4} \epsilon^{\mu \nu \rho \sigma} F_{\mu \nu} F_{\rho \sigma} \\
I_{\mathrm{GB}} & =\alpha \int_{M} d^{4} x \sqrt{-g}\left(R^{2}-4 R^{\mu \nu} R_{\mu \nu}+R^{\mu \nu \rho \sigma} R_{\mu \nu \rho \sigma}\right) .
\end{aligned}
$$


Both terms are expected to have electromagnetic duality, in the type of minimally-coupled (generalized) Maxwell theories we consider in this paper.

Finally, it should be pointed out that equations (2.2) and (2.4) interchange each other if we interchange $* F$ and $\widetilde{F}$, indicating the electromagnetic duality can always be implemented for the equations. However, the energy-momentum tensor expressed in $F$ and $\widetilde{F}$, namely $T_{\mu \nu}(F)$ and $T_{\mu \nu}(\widetilde{F})$, may not have the same form and hence the electromagnetic duality can be broken by the Einstein equation. In this paper, we shall only consider examples where the electromagnetic duality is indeed the symmetry for the equations and the metric of the dyonic black hole depends only on the combination $Q_{e}^{2}+Q_{m}^{2}$.

\section{Einstein-Maxwell thoery}

In this section, we review the Einstein-Maxwell theory to illustrate a feature that the electric and magnetic contributions to the holographic complexity of a dyonic black hole are different and how this can be resolved [44]. Einstein-Maxwell theory in four dimensions, including the Gibbons-Howking term, is given by

$$
I=\frac{\kappa}{16 \pi} \int_{M} d^{4} x \sqrt{-g}\left(R-2 \Lambda-\frac{1}{4} F^{2}\right)+\frac{\kappa}{8 \pi} \int_{\partial M} d^{3} x \sqrt{-h} K,
$$

where $h$ is the determinant of the boundary induced metric, $K$ is the trace of the extensive tensor and we set $\Lambda=-3 / \ell^{2}$. The theory admits the dyonic Reissner-Nordström-AdS (RN-AdS) blakc hole.

$$
d s^{2}=-f d t^{2}+\frac{d r^{2}}{f}+r^{2}\left(d \theta^{2}+\sin ^{2} \theta d \phi^{2}\right), \quad A=a d t+p \cos \theta d \phi,
$$

with

$$
f=\frac{r^{2}}{\ell^{2}}+1-\frac{\mu}{r}+\frac{q^{2}+p^{2}}{4 r^{2}}, \quad a=\frac{q}{r}
$$

Here $(\mu, p, q)$ are integration constants. We choose the gauge that the electric potential $a$ vanishes at infinity. The mass of the black hole, electric charge, magnetic charge, electric potential and magnetic potentials are

$$
M=\frac{\kappa \mu}{2}, \quad Q_{e}=\frac{\kappa q}{4}, \quad Q_{m}=\frac{\kappa p}{4}, \quad \Phi_{e \pm}=\frac{q}{r_{ \pm}}, \quad \Phi_{m \pm}=\frac{p}{r_{ \pm}},
$$

where \pm represent quantities evaluated on the outer and inner horizons of the black hole.

In order to calculate the late-time action growth, we follow the method in [13] and we shall present only the main results. For more details we refer to [13]. To apply this method, we introduce the null coordinates $(u, v)$, defined by

$$
d u=d t+\frac{d r}{\sqrt{h f}}, \quad d v=d t-\frac{d r}{\sqrt{h f}} .
$$

The Wheeler-De Witt patch of the black hole is shown in figure 1, which is surrounded by the light rays symmetrically anchored to the left and right boundary time slices with $t_{L}=t_{R}=t / 2$. The action growth rate consists of three parts (bulk, boundary and joint),

$$
\frac{d I}{d t}=\frac{d I_{\mathrm{bulk}}}{d t}+\frac{d I_{\mathrm{bd}}}{d t}+\frac{d I_{\mathrm{joint}}}{d t}
$$


with

$$
\begin{aligned}
\frac{d I_{\mathrm{bulk}}}{d t} & =\left.\frac{\kappa}{4}\left(\frac{\left(p^{2}-q^{2}\right)}{2 r}-2 g^{2} r^{3}\right)\right|_{r_{-}} ^{r_{+}}, \\
\frac{d}{d t}\left(I_{\text {joint }}+I_{\mathrm{bd}}\right) & =\left.\frac{\kappa}{4}\left(-\frac{\left(p^{2}+q^{2}\right)}{2 r}+2 g^{2} r^{3}\right)\right|_{r_{-}} ^{r_{+}} .
\end{aligned}
$$

Thus we see that the magnetic and electric contributions have opposite signs in the first term of the bulk contribution, originated from the kinetic $F^{2}$ term. There are cancelations among these parts, so that the total action growth rate is quite simple

$$
\frac{d I}{d t}=\frac{d I_{\mathrm{bulk}}}{d t}+\frac{d}{d t}\left(I_{\mathrm{joint}}+I_{\mathrm{ct}}\right)=-\left.\frac{\kappa q^{2}}{4 r}\right|_{r_{-}} ^{r_{+}}=-\left.\Phi_{e} Q_{e}\right|_{r_{-}} ^{r_{+}} .
$$

The result is independent of magnetic charge, in other words, the electric charge and the magnetic charge are not in the equal footing. In particular, the action vanishes for the purely magnetic black hole. It is worth commenting that this is a special result of a more general formula

$$
\frac{d I}{d t}=(F+T S)_{+}-(F+T S)_{-}=\mathcal{H}_{+}-\mathcal{H}_{-},
$$

shown in [28] for all the black holes with both inner and outer horizons.

To address this issue, it was pointed out that at the price of introducing mixed boundary conditions in the variation principle, one can add a boundary term for the Maxwell field with an arbitrary coefficient $\gamma[44]$

$$
\left.I_{\mu Q}=\frac{\kappa \gamma}{16 \pi} \int_{\partial M} d \Sigma_{\mu} F^{\mu \nu} A_{\nu}, \quad \text { (i.e. } \quad \widetilde{F}^{\mu \nu}=F^{\mu \nu}\right) .
$$

On shell, with the help of the Maxwell equation and the Stokes' theorem, this term can be written as a bulk integration

$$
\left.I_{\mu Q}\right|_{\text {on-shell }}=\frac{\kappa \gamma}{32 \pi} \int_{M} d^{4} x \sqrt{-g} F^{2} .
$$

Thus on shell, the total contributions of the Maxwell field to the action is modified to become

$$
I_{\mathrm{Max}}+\left.I_{\mu Q}\right|_{\text {on-shell }}=\frac{(2 \gamma-1) \kappa}{64 \pi} \int_{M} d^{4} x \sqrt{-g} F^{2} .
$$

Thus after including the boundary term, the total action growth rate in the WDW patch is now given by

$$
\frac{d I}{d t}=\left.\left(-(1-\gamma) \Phi_{e} Q_{e}-\gamma \Phi_{m} Q_{m}\right)\right|_{r_{-}} ^{r_{+}}
$$

As can be expected, the action growth has electromagnetic duality when $\gamma=\frac{1}{2}$.

At the end of section 2, two more total derivative terms were mentioned. The GaussBonnet term gives no contribution to the action growth for black holes with two horizons. We find that the $F \wedge F$-term gives

$$
\frac{d I_{F \wedge F}}{d t}=-\left.\frac{\tilde{\gamma} \kappa p q}{2 r}\right|_{r_{-}} ^{r_{+}} .
$$


It is clear that the action growth has electromagnetic duality. Compared to (3.13), it should perhaps be expressed as

$$
\frac{d I_{F \wedge F}}{d t}=-\left.\tilde{\gamma}\left(\Phi_{e} Q_{m}+\Phi_{m} Q_{e}\right)\right|_{r_{-}} ^{r_{+}}
$$

Thus the most general form of the action growth rate that has the electromagnetic duality is

$$
\frac{d I}{d t}=\left.\left(-\frac{1}{2}\left(\Phi_{e} Q_{e}+\Phi_{m} Q_{m}\right)-\tilde{\gamma}\left(\Phi_{e} Q_{m}+\Phi_{m} Q_{e}\right)\right)\right|_{r_{-}} ^{r_{+}},
$$

where the coefficient $\tilde{\gamma}$ can be arbitrary.

\section{Einstein-Maxwell-Dilaton theory}

In [44], the authors presented the complexity of a purely electric (or magnetic) AdS black hole in a class of EMD theories [53]. In this section, we go one step further and investigate AdS dyonic black hole solutions in certain EMD theories. We consider

$$
\mathcal{L}=\sqrt{-g}\left(R-\frac{1}{2}(\partial \varphi)^{2}-\frac{1}{4} e^{a \varphi} F^{2}-V(\varphi)\right), \quad F=d A
$$

The scalar potential can be expressed in terms of a superpotential $W$ [54]

$$
V=\left(\frac{d W}{d \varphi}\right)^{2}-\frac{3}{4} W^{2}, \quad W=\frac{2 \sqrt{2} g}{a^{2}+1}\left(e^{-\frac{1}{2} a \varphi}+a^{2} e^{\frac{1}{2 a} \varphi}\right) .
$$

Explicitly, the scalar potential is [53]

$$
V=-\frac{2 g^{2}}{\left(1+a^{2}\right)^{2}}\left[a^{2}\left(3 a^{2}-1\right) e^{\frac{\varphi}{a}}+\left(3-a^{2}\right) e^{-a \varphi}+8 a^{2} e^{\left(-\frac{a}{2}+\frac{1}{2 a}\right) \varphi}\right] .
$$

Note that taking the Taylor series expansion in the region of $\varphi=0$, we have

$$
V=-6 g^{2}-g^{2} \varphi^{2}+\frac{a^{4}-4 a^{2}+1}{24 a^{2}} g^{2} \varphi^{4}-\frac{\left(a^{2}-\frac{1}{3}\right)\left(a^{2}-1\right)\left(a^{2}-3\right)}{80 a^{3}} g^{2} \varphi^{5}+\cdots .
$$

Thus the limit of $a=0$ requires that $\varphi=0$. It follows from the kinetic term of the Maxwell field that the electromagnetic duality in the EMD theory requires

$$
e^{a \varphi} * F \quad \leftrightarrow \quad F, \quad \varphi \quad \leftrightarrow \quad-\varphi
$$

The scalar potential $V$ breaks this duality except when $a=1 / \sqrt{3}, 1$ and $\sqrt{3}$, for which $V(\varphi)=V(-\varphi)$. Exact solutions of charged AdS dyonic black holes were known only for the latter two cases. Thus we study the holographic complexity for $a=1$ and $a=\sqrt{3}$ dyonic black holes.

In order to recover the electromagnetic duality in the late-time action growth, a Maxwell boundary term is introduced [44]

$$
\left.I_{\mu Q}=\frac{\kappa \gamma}{16 \pi} \int_{\partial M} d \Sigma_{\mu} e^{a \varphi} F^{\mu \nu} A_{\nu}, \quad \text { (i.e. } \quad \widetilde{F}^{\mu \nu}=e^{a \varphi} F^{\mu \nu}\right)
$$

As in the previous case, the on-shell action can be written as a bulk integratoin

$$
\left.I_{\mu Q}\right|_{\text {on-shell }}=\frac{\kappa \gamma}{32 \pi} \int_{M} d^{4} x \sqrt{-g} e^{-a \varphi} F^{2} .
$$




\subsection{Dyonic black hole with $a=1$}

When $a=1$ the scalar potential can be written explicitly as

$$
V=-2 g^{2}(2+\cosh \varphi)
$$

The dyonic black hole solution is [54]

$$
\begin{aligned}
d s^{2} & =-\left(H_{1} H_{2}\right)^{-1} f d t^{2}+H_{1} H_{2}\left(\frac{d r^{2}}{f}+r^{2}\left(d \theta^{2}+\sin ^{2} \theta d \phi^{2}\right)\right), \\
A & =\sqrt{2} c_{1} s_{1}^{-1} H_{1}^{-1} d t+\sqrt{2} c_{2} s_{2} \cos \theta d \phi, \\
\varphi & =\log \left(H_{1} / H_{2}\right), \quad H_{i}=1+\frac{\mu s_{i}^{2}}{r}, \quad f=1-\frac{\mu}{r}+g^{2} r^{2} H_{1}^{2} H_{2}^{2},
\end{aligned}
$$

where $c_{i}=\cosh \delta_{i}$ and $s_{i}=\sinh \delta_{i}$. The mass, electric charge, electric potential, magnetic charge and potential are given by

$$
\begin{aligned}
M & =\frac{1}{2} \mu\left(1+s_{1}^{2}+s_{2}^{2}\right), & Q_{e} & =\frac{1}{4} \sqrt{2} \mu c_{1} s_{1},
\end{aligned} Q_{M}=\frac{1}{4} \sqrt{2} \mu c_{2} s_{2},
$$

where $r_{ \pm}$are the radius of the outer and inner horizon of the black hole.

Including the Maxwell boundary term (4.6), the action growth rate can be written as

$$
\frac{d I}{d t}=\frac{d\left(I_{\mathrm{bulk}}+I_{\mu Q}\right)}{d t}+\frac{d I_{\mathrm{bd}}}{d t}+\frac{d I_{\text {joint }}}{d t}
$$

with

$$
\begin{aligned}
& \frac{d\left(I_{\mathrm{bulk}}+I_{\mu Q}\right)}{d t}=\left.\left(\frac{(r-\mu)\left(r^{2}-\mu^{2} s_{1}^{2} s_{2}^{2}\right)}{2\left(r+\mu s_{1}^{2}\right)\left(r+\mu s_{2}^{2}\right)}-(1-\gamma) \Phi_{e} Q_{e}-\gamma \Phi_{m} Q_{m}\right)\right|_{r_{-}} ^{r_{+}}, \\
& \frac{d\left(I_{\mathrm{bd}}+I_{\text {joint }}\right)}{d t}=-\left.\frac{(r-\mu)\left(r^{2}-\mu^{2} s_{1}^{2} s_{2}^{2}\right)}{2\left(r+\mu s_{1}^{2}\right)\left(r+\mu s_{2}^{2}\right)}\right|_{r_{-}} ^{r_{+}} .
\end{aligned}
$$

The total action growth rate takes the identical form (3.13) as the Einstein-Maxwell theory.

\subsection{Dyonic black hole with $a=\sqrt{3}$}

The case of $a=\sqrt{3}$ is the well-known Kuluza-Klein (KK) theory with the scalar potential

$$
V=-6 g^{2} \cosh \left(\frac{1}{\sqrt{3}} \varphi\right)
$$


The KK dyonic AdS black hole was constructed in [52]. For simplicity, we consider only the planar geometry, for which the solution is

$$
\begin{array}{rlrl}
d s^{2} & =-\left(H_{1} H_{2}\right)^{-\frac{1}{2}} f d t^{2}+\left(H_{1} H_{2}\right)^{\frac{1}{2}}\left(\frac{d r^{2}}{f}+r^{2}\left(d x^{2}+d y^{2}\right)\right), \\
\varphi & =\frac{\sqrt{3}}{2} \log \frac{H_{2}}{H_{1}}, & f=-\frac{2 \mu}{r}+g^{2} r^{2} H_{1} H_{2}, \\
A & =\sqrt{2 \mu}\left(\frac{r+2 \beta_{1}}{\sqrt{\beta_{1}} H_{1} r} d t+2 \sqrt{\beta_{2}} x d y\right), & \\
H_{1} & =1+\frac{4 \beta_{1}}{r}+\frac{4 \beta_{1} \beta_{2}}{r^{2}}, & H_{2} & =1+\frac{4 \beta_{2}}{r}+\frac{4 \beta_{1} \beta_{2}}{r^{2}} .
\end{array}
$$

The solution has integration constants $\left(\mu, \beta_{1}, \beta_{2}\right)$, parameterizing the mass and electric and magnetic charges:

$$
M=\mu, \quad Q_{e}=\sqrt{\frac{\mu \beta_{1}}{2}}, \quad Q_{m}=\sqrt{\frac{\mu \beta_{2}}{2}},
$$

The electric and magnetic potentials on both inner and outer horizons $r_{ \pm}$are

$$
\Phi_{e \pm}=\left.\frac{2 \sqrt{2 \mu \beta_{1}}\left(r+2 \beta_{2}\right)}{r^{2} H_{1}}\right|_{ \pm}, \quad \Phi_{m \pm}=\left.\frac{2 \sqrt{2 \mu \beta_{2}}\left(r+2 \beta_{1}\right)}{r^{2} H_{2}}\right|_{ \pm},
$$

Including the Maxwell boundary term (4.6), the action growth rate is (4.11) and we find

$$
\begin{aligned}
\frac{d\left(I_{\mathrm{bulk}}+I_{\mu Q}\right)}{d t}= & \left(-(1-\gamma) \Phi_{e} Q_{e}-\gamma \Phi_{m} Q_{m}\right. \\
& \left.-\frac{\mu\left(2 \beta_{1}+r\right)\left(2 \beta_{2}+r\right)\left(r^{2}-4 \beta_{1} \beta_{2}\right)}{\left(4 \beta_{1} \beta_{2}+r^{2}+4 \beta_{1} r\right)\left(4 \beta_{1} \beta_{2}+r^{2}+4 \beta_{2} r\right)}\right)\left.\right|_{r_{-}} ^{r_{+}} \\
\frac{d\left(I_{\mathrm{bd}}+I_{\text {joint }}\right)}{d t}= & \left.\frac{\mu\left(2 \beta_{1}+r\right)\left(2 \beta_{2}+r\right)\left(r^{2}-4 \beta_{1} \beta_{2}\right)}{\left(4 \beta_{1} \beta_{2}+r^{2}+4 \beta_{1} r\right)\left(4 \beta_{1} \beta_{2}+r^{2}+4 \beta_{2} r\right)}\right|_{r_{-}} ^{r_{+}}
\end{aligned}
$$

The total action growth rate is again (3.13). Thus we see that the action growth rate of dyonic AdS black holes in EMD theories take the same form as that of Einstein-Maxwell theory, and it recovers the electromagnetic duality when $\gamma=\frac{1}{2}$.

\section{Einstein-Born-Infeld theory}

The theory of Einstein-Born-Infeld (EBI) is a generalization of the Born-Infeld (BI) theory [55] to include gravity. The Lagrangian, with a bare cosmological constant $\Lambda_{0}$, is given by

$$
\mathcal{L}=\sqrt{g}\left(R-2 \Lambda_{0}\right)-b^{2} \sqrt{-\operatorname{det}\left(g_{\mu \nu}+\frac{F_{\mu \nu}}{b}\right)}
$$


where $\Lambda_{0}=\Lambda-\frac{b^{2}}{2}$ and $\Lambda$ is the effective cosmological constant. In four dimensions, the theory admits AdS dyonic black hole solutions [56]

$$
d s^{2}=-f d t^{2}+\frac{d r^{2}}{f}+r^{2}\left(d \theta^{2}+\sin ^{2} \theta d \phi^{2}\right), \quad A=a d t+p \cos \theta d \phi,
$$

with

$$
\begin{aligned}
& f=-\frac{1}{3} \Lambda_{0} r^{2}+1-\frac{\mu}{r}-\frac{b^{2}}{6} \sqrt{r^{4}+\frac{Q^{2}}{b^{2}}}+\frac{Q^{2}}{3 r^{2}}{ }_{2} F_{1}\left[\frac{1}{4}, \frac{1}{2} ; \frac{5}{4} ;-\frac{Q^{2}}{b^{2} r^{4}}\right], \\
& a=\frac{q}{r}{ }_{2} F_{1}\left[\frac{1}{4}, \frac{1}{2} ; \frac{5}{4} ;-\frac{Q^{2}}{b^{2} r^{4}}\right], \quad Q^{2}=p^{2}+q^{2},
\end{aligned}
$$

where $(\mu, p, q)$ are the integration constants parameterizing the mass and charges of the black hole. To be specific, we have

$$
\begin{aligned}
M & =\frac{\mu}{2}, & Q_{e} & =\frac{q}{4},
\end{aligned} Q_{m}=\frac{p}{4},
$$

where $r_{ \pm}$are the radius of the outer and inner horizons. Thus we see that the metric, depending on $Q_{e}^{2}+Q_{m}^{2}$, has the electromagnetic duality.

Although the Maxwell field is singularity free, the black hole solution has a curvature singularity at $r=0$. When $\sqrt{p^{2}+q^{2}} \leq 2 / b$, or $\sqrt{p^{2}+q^{2}}>2 / b$ but with $M \geq M^{*}$, the dyonic black hole has only one event horizon, where

$$
M^{*}=\frac{\Gamma\left(\frac{1}{4}\right)^{2} \sqrt{b}}{24 \sqrt{\pi}} Q^{\frac{3}{2}}
$$

When $\sqrt{p^{2}+q^{2}}>2 / b$ and $M_{\text {extrem }}<M<M^{*}$, the solution has two horizons. More details about the structure of the black hole singularity and the black hole thermodynamics can be found in [57].

We are now in the stage of calculating the on-shell action growth rate. As discussed in section 2, now the Maxwell boundary term is given by

$$
I_{\mu Q}=\gamma \frac{1}{16 \pi} \int_{\partial M} d \Sigma_{\mu} \widetilde{F}^{\mu \nu} A_{\nu}, \quad \widetilde{F}^{\mu \nu}=-b \frac{\sqrt{-h}}{\sqrt{-g}}\left(h^{-1}\right)^{[\mu \nu]},
$$

where

$$
h_{\mu \nu}=g_{\mu \nu}+\frac{F_{\mu \nu}}{b}, \quad \sqrt{h}=\sqrt{-h_{\mu \nu}}, \quad\left(h^{-1}\right)^{[\mu \nu]}=\frac{1}{2}\left(\left(h^{-1}\right)^{\mu \nu}-\left(h^{-1}\right)^{\nu \mu}\right),
$$

and $\left(h^{-1}\right)^{\mu \nu}$ is the inverse of $h_{\mu \nu}$. (There should be no confusion between the notation of this $h_{\mu \nu}$ and the boundary induced metric $h_{a b}$ in the Gibbons-Hawking surface term.) The on-shell action can be expressed in terms of the bulk integration

$$
I_{\mathrm{on}-\mathrm{shell}}^{\mu Q}=-\frac{1}{16 \pi} \int_{M} d^{4} x \frac{1}{2} \gamma b \sqrt{h}\left(h^{-1}\right)^{[\mu \nu]} F_{\mu \nu} .
$$


Having included this term, the total action growth rate is again given by (4.11), but with

$$
\begin{aligned}
\frac{d\left(I_{\mathrm{bulk}}+I_{\mu Q}\right)}{d t}= & \left(-(1-\gamma) \Phi_{e} Q_{e}-\gamma \Phi_{m} Q_{m}\right. \\
& \left.+\frac{1}{8} b r \sqrt{p^{2}+q^{2}+b^{2} r^{4}}-\frac{r}{4}+\frac{\Lambda}{4} r^{3}\right)\left.\right|_{r_{-}} ^{r_{+}}, \\
\frac{d\left(I_{\mathrm{bd}}+I_{\text {joint }}\right)}{d t}= & \left.\left(-\frac{1}{8} b r \sqrt{p^{2}+q^{2}+b^{2} r^{4}}+\frac{r}{4}-\frac{\Lambda}{4} r^{3}\right)\right|_{r_{-}} ^{r_{+}} .
\end{aligned}
$$

Thus the later-time action growth rate again takes the same form as the Einstein-Maxwell case, given by (3.13).

For the dyonic black holes with only one horizon, the causal structure of the black hole is analogous to that of the Schwarzschild black hole. We follow the same procedure [13] and find

$$
\begin{aligned}
\frac{d I}{d t}= & 2 M-(1-\gamma) \Phi_{e+} Q_{e}-\gamma \Phi_{m+} Q_{m} \\
& -\frac{5 \sqrt{\beta} \Gamma\left(\frac{1}{4}\right) \Gamma\left(\frac{5}{4}\right)\left(Q_{e}^{2}+Q_{m}^{2}\right)^{3 / 4}}{3 \sqrt{\pi}}+\frac{\sqrt{\beta}(1-2 \gamma) \Gamma\left(\frac{1}{4}\right) \Gamma\left(\frac{5}{4}\right)\left(Q_{e}^{2}-Q_{m}^{2}\right)}{\sqrt{\pi} \sqrt[4]{Q_{e}^{2}+Q_{m}^{2}}}
\end{aligned}
$$

The result contains two parts. The first line of the above is related to the black hole event horizon and can be expressed in terms of thermodynamical quantities. The second line of the formula comes from the contribution of the curvature singularity $r=0$. Both parts independently contain the electromagnetic duality when $\gamma=\frac{1}{2}$.

The emergency of the electromagnetic duality at precise $\gamma=\frac{1}{2}$ is much less trivial than the previous cases. This is because now the duality-violating term (2.5) no longer simply vanish when $\gamma=\frac{1}{2}$. In fact, the on-shell Maxwell bulk contribution is given by

$$
\begin{aligned}
\frac{d}{d t}\left(\frac{-b^{2}}{16 \pi} \int_{M} d^{4} x \sqrt{-\operatorname{det}\left(g_{\mu \nu}+\frac{F_{\mu \nu}}{b}\right)}\right) & \\
= & \frac{1}{4}\left(\frac{1}{3} b r \sqrt{16\left(Q_{e}^{2}+Q_{m}^{2}\right)+b^{2} r^{4}}-\frac{2 b r\left(Q_{e}^{2}+Q_{m}^{2}\right){ }_{2} F_{1}\left[\frac{1}{4}, \frac{1}{2} ; \frac{5}{4} ;-\frac{r^{4} b^{2}}{16\left(Q_{e}^{2}+Q_{m}^{2}\right)}\right]}{3 \sqrt{Q_{e}^{2}+Q_{m}^{2}}}\right. \\
& \left.+\frac{2 b r\left(Q_{e}^{2}-Q_{m}^{2}\right){ }_{2} F_{1}\left[\frac{1}{4}, \frac{1}{2} ; \frac{5}{4} ;-\frac{r^{4} b^{2}}{16\left(Q_{e}^{2}+Q_{m}^{2}\right)}\right]}{\sqrt{Q_{e}^{2}+Q_{m}^{2}}}\right)\left.\right|_{r_{-}} ^{r_{+}}
\end{aligned}
$$

while the Maxwell boundary term on-shell is given by

$$
\frac{d \mathcal{I}_{\mu Q}}{d t}=-\left.\frac{b \gamma r\left(Q_{e}^{2}-Q_{m}^{2}\right){ }_{2} F_{1}\left[\frac{1}{4}, \frac{1}{2} ; \frac{5}{4} ;-\frac{r^{4} b^{2}}{16\left(Q_{e}^{2}+Q_{m}^{2}\right)}\right]}{\sqrt{Q_{e}^{2}+Q_{m}^{2}}}\right|_{r_{-}} ^{r_{+}} .
$$


Together, the total Maxwell contribution is

$$
\begin{aligned}
\frac{d I_{F}}{d t}= & \frac{1}{4}\left(\frac{1}{3} b r \sqrt{16\left(Q_{e}^{2}+Q_{m}^{2}\right)+b^{2} r^{4}}-\frac{2 b r\left(Q_{e}^{2}+Q_{m}^{2}\right){ }_{2} F_{1}\left[\frac{1}{4}, \frac{1}{2} ; \frac{5}{4} ;-\frac{r^{4} b^{2}}{16\left(Q_{e}^{2}+Q_{m}^{2}\right)}\right]}{3 \sqrt{Q_{e}^{2}+Q_{m}^{2}}}\right. \\
& \left.+\frac{2(1-2 \gamma) b r\left(Q_{e}^{2}-Q_{m}^{2}\right){ }_{2} F_{1}\left[\frac{1}{4}, \frac{1}{2} ; \frac{5}{4} ;-\frac{r^{4} b^{2}}{16\left(Q_{e}^{2}+Q_{m}^{2}\right)}\right]}{\sqrt{Q_{e}^{2}+Q_{m}^{2}}}\right)\left.\right|_{r_{-}} ^{r_{+}}
\end{aligned}
$$

We can see that when $\gamma=1 / 2$, the Maxwell boundary term cancels the asymmetric part of the bulk contribution, which is proportional to $Q_{e}^{2}-Q_{m}^{2}$. What is left is symmetric with $\left(Q_{e}, Q_{m}\right)$ that appears as $Q_{e}^{2}+Q_{m}^{2}$ in the on-shell action.

\section{Einstein-Maxwell-Horndeski theory}

Einstein-Horndeski theory is a type of higher-derivative scalar-tensor theory where each field has no more than two derivatives in the equations of motion [58]. This feature is similar to that of Lovelock gravity [59]. A class of black hole solutions were constructed in Horndeski theory in [60,61] and their thermodynamics were studied in [62, 63], the instability and holographic applications can be found in [64-76] and reference therein. In particular it was proposed that the theory is a holographic dual to some scale invariant but not conformal invariant quantum field theory $[74,76]$. The theory we consider is given by

$$
\mathcal{S}=\frac{1}{16 \pi} \int_{M} \sqrt{-g}\left[\kappa\left(R-2 \Lambda-\frac{1}{4} F^{2}\right)-\frac{1}{2}\left(\alpha g^{\mu \nu}-\eta G^{\mu \nu}\right) \partial_{\mu} \chi \partial_{\nu} \chi\right]
$$

where $G_{\mu \nu}=R_{\mu \nu}-\frac{1}{2} R g_{\mu \nu}$ is the Einstein tensor, $\chi$ is the scalar field. It admits static AdS black hole solutions with both planar topology $\epsilon=0$ and spherical topology $\epsilon=1$ [61].

\subsection{Charged AdS planar black hole}

The charged AdS planar black hole is given by

$$
\begin{aligned}
d s^{2} & =-h(r) d t^{2}+\frac{d r^{2}}{f(r)}+r^{2}\left(d x_{1}^{2}+d x_{2}^{2}\right), \\
A & =a(r) d t+\frac{1}{2} p\left(x_{1} d x_{2}-x_{2} d x_{1}\right), \quad \chi=\chi(r),
\end{aligned}
$$

we find that the theory admits a black hole solution

$$
\begin{aligned}
f & =\frac{36 g^{4} r^{8}(\beta \eta+4 \kappa)^{2}}{\left(\kappa\left(q^{2}+p^{2}\right)-6 g^{2} r^{4}(\beta \eta+4 \kappa)\right)^{2}} h, \\
h & =g^{2} r^{2}-\frac{\mu}{r}+\frac{\kappa\left(q^{2}+p^{2}\right)}{r^{2}(\beta \eta+4 \kappa)}-\frac{\kappa^{2}\left(q^{2}+p^{2}\right)^{2}}{60 g^{2} r^{6}(\beta \eta+4 \kappa)^{2}}, \\
\chi^{\prime} & =\sqrt{\beta-\frac{\kappa\left(q^{2}+p^{2}\right)}{6 \eta g^{2} r^{4}}} \sqrt{\frac{1}{f}}, \quad a=-\frac{q}{r}+\frac{\kappa q\left(q^{2}+p^{2}\right)}{30 g^{2} r^{5}(\beta \eta+4 \kappa)},
\end{aligned}
$$


with constraints

$$
\Lambda=-\frac{3 g^{2}(\beta \eta+2 \kappa)}{2 \kappa}, \quad \alpha=3 \eta g^{2} .
$$

The global structure and black hole thermodynamics was analysed in [63]. Here we give some relevant thermodynamical quantities

$$
\begin{array}{ll}
M=\frac{4 \kappa+\beta \eta}{32 \pi} \mu, & T=-\left.\frac{\kappa\left(p^{2}+q^{2}\right)-6 g^{2} r^{4}(\beta \eta+4 \kappa)}{8 \pi r^{3}(\beta \eta+4 \kappa)}\right|_{r_{0}}, \\
\Phi_{q}=-\left.\frac{q\left(\kappa\left(p^{2}+q^{2}\right)-30 g^{2} r^{4}(\beta \eta+4 \kappa)\right)}{30 g^{2} r^{5}(\beta \eta+4 \kappa)}\right|_{r_{0}}, & Q_{q}=\frac{\kappa q}{16 \pi}, \\
\Phi_{p}=-\left.\frac{p\left(\kappa\left(p^{2}+q^{2}\right)-30 g^{2} r^{4}(\beta \eta+4 \kappa)\right)}{30 g^{2} r^{5}(\beta \eta+4 \kappa)}\right|_{r_{0}}, & Q_{p}=\frac{\kappa p}{16 \pi} .
\end{array}
$$

Different from the RN-AdS black hole, there is an additional singularity $r_{*}$ where $f$ diverges

$$
\kappa\left(p^{2}+q^{2}\right)-6 g^{2} r_{*}^{4}(\beta \eta+4 \kappa)=0 .
$$

There is a crucial difference with the RN black hole, this black hole has only one event horizon and thus has no extremal limit. Even though the temperature can be arbitrarily small, it can never reach zero [36, 62, 63].

Now, we are in the position of calculating the action growth. Including the Maxwell boundary $\gamma$-term, we find that the total action growth is

$$
\begin{aligned}
\frac{d I}{d t}= & 2 M-(1-\gamma) \Phi_{e} Q_{e}-\gamma \Phi_{m} Q_{m} \\
& -\frac{32 \pi\left(Q_{e}^{2}+Q_{m}^{2}\right)}{3 \kappa r_{*}}+\frac{32 \pi(1-2 \gamma)\left(Q_{e}^{2}-Q_{m}^{2}\right)}{5 \kappa r_{*}} .
\end{aligned}
$$

\subsection{Charged AdS spherical black hole}

The solution is given by

$$
\begin{gathered}
d s^{2}=-h(r) d t^{2}+\frac{d r^{2}}{f(r)}+r^{2}\left(d \theta^{2}+\sin \theta^{2} d \phi^{2}\right), \\
A=a(r) d t+p \cos \theta d \phi, \quad \chi=\chi(r),
\end{gathered}
$$

with

$$
\begin{aligned}
f= & \frac{4 r^{4}\left(3 g^{2} r^{2}+1\right)^{2}(\beta \eta+4 \kappa)^{2}}{\left(\kappa\left(q^{2}+p^{2}-8 r^{2}\right)-6 g^{2} r^{4}(\beta \eta+4 \kappa)\right)^{2}} h, \\
h= & \frac{4 \kappa-\beta \eta}{\beta \eta+4 \kappa}+\frac{\kappa^{2}\left(p^{2}+q^{2}\right)\left(3 p^{2} g^{2}+3 g^{2} q^{2}+16\right)}{4 r^{2}(\beta \eta+4 \kappa)^{2}}-\frac{\kappa^{2}\left(p^{2}+q^{2}\right)^{2}}{12 r^{4}(\beta \eta+4 \kappa)^{2}}+g^{2} r^{2}-\frac{\mu}{r} \\
& -\frac{\tan ^{-1}\left(\frac{1}{\sqrt{3} g r}\right)\left(2 \beta \eta-3 g^{2} \kappa\left(p^{2}+q^{2}\right)\right)^{2}}{4 \sqrt{3} g r(\beta \eta+4 \kappa)^{2}}
\end{aligned}
$$




$$
\begin{aligned}
\chi^{\prime}= & \sqrt{\frac{6 \beta \eta g^{2} r^{4}-\kappa\left(q^{2}+p^{2}\right)}{2 \eta r^{2}\left(1+3 g^{2} r^{2}\right)}} \sqrt{\frac{1}{f}}, \\
a= & -\frac{\kappa q\left(3 g^{2}\left(q^{2}+p^{2}\right)+8\right)}{2 r(\beta \eta+4 \kappa)}+\frac{\kappa q\left(q^{2}+p^{2}\right)}{6 r^{3}(\beta \eta+4 \kappa)} \\
& +\frac{\sqrt{3} g q \tan ^{-1}\left(\frac{1}{\sqrt{3} g r}\right)\left(3 g^{2} \kappa\left(q^{2}+p^{2}\right)-2 \beta \eta\right)}{2(\beta \eta+4 \kappa)},
\end{aligned}
$$

together with the constraints

$$
\Lambda=-\frac{3 g^{2}(\beta \eta+2 \kappa)}{2 \kappa}, \quad \alpha=3 \eta g^{2}
$$

To be precise, the black hole is asymptotically locally AdS. The global structure and black hole thermodybamics was analysed in [63]. The relevant thermodynamical quantities are

$$
M=\frac{4 \kappa+\beta \eta}{8} \mu, \quad Q_{e}=\frac{\kappa q}{4}, \quad Q_{m}=\frac{\kappa p}{4}, \quad \Phi_{e}=\frac{\xi q}{r_{+}}, \quad \Phi_{m}=\frac{\xi p}{r_{+}},
$$

where

$$
\xi=\frac{\kappa\left(24+\left(p^{2}+q^{2}\right)\left(9 g^{2}-\frac{1}{r_{+}^{2}}\right)\right)-3 \sqrt{3} g r_{+} \tan ^{-1}\left(\frac{1}{\sqrt{3} g r_{+}}\right)\left(3 g^{2} \kappa\left(p^{2}+q^{2}\right)-2 \beta \eta\right)}{6(\beta \eta+4 \kappa)},
$$

The black hole has only one horizon and there is a curvature singularity at $r_{*}$ where $f$ diverges, namely

$$
\kappa\left(q^{2}+p^{2}-8 r_{*}^{2}\right)-6 g^{2} r_{*}^{4}(\beta \eta+4 \kappa)=0 .
$$

We find that the total action growth (including the Maxwell boundary term) is given by

$$
\begin{aligned}
\frac{d I}{d t}= & 2 M-(1-\gamma) \Phi_{e} Q_{e}-\gamma \Phi_{m} Q_{m} \\
& -\frac{1}{4} g^{2} r_{*}^{3}(\beta \eta+4 \kappa)+r_{*}\left(\frac{\beta \eta}{4}-\kappa\right)-\frac{8\left(Q_{e}^{2}+Q_{m}^{2}\right)}{r_{*}(\beta \eta+4 \kappa)} \\
& +\frac{\beta \eta \tan ^{-1}\left(\frac{1}{\sqrt{3} g r_{*}}\right)\left(\beta \eta \kappa-24 g^{2}\left(Q_{e}^{2}+Q_{m}^{2}\right)\right)}{4 \sqrt{3} g \kappa(\beta \eta+4 \kappa)} \\
& +\frac{2(1-2 \gamma)\left(Q_{e}^{2}-Q_{m}^{2}\right)}{3 \kappa^{2} r_{*}^{3}(\beta \eta+4 \kappa)}\left(3 \sqrt{3} g r_{*}^{3} \tan ^{-1}\left(\frac{1}{\sqrt{3} g r_{*}}\right)\left(\beta \eta \kappa-24 g^{2} Q_{p}^{2}-24 g^{2} Q_{q}^{2}\right)\right. \\
& \left.+8\left(9 g^{2} r_{*}^{2}-1\right)\left(Q_{p}^{2}+Q_{q}^{2}\right)+12 \kappa^{2} r_{*}^{2}\right) .
\end{aligned}
$$

Thus although the results appear to be more complicated, but it maintains the essential feature, namely the action growth rate preserves the electromagnetic duality when $\gamma=\frac{1}{2}$. 


\section{Conclusions}

In this paper, we investigated the holographic complexity for dyonic black holes in EinsteinMaxwell-Dilaton theory, Einstein-Born-Infeld theroy and Einstein-Maxwell-Horndeski theory by means of the CA conjecture. The theories all have the electromagnetic duality, but realized only at the level of equations. We presented a universal $\gamma$-term in both first-order and second-order formalism so that the duality was restored in the on-shell action. The advantage of the first-order formalism is that the variation principle involves only the standard Dirichlet boundary condition instead of the mixed boundary condition required by the second-order formalism.

For the dyonic black holes with either two horizons or just one horizon, we find that the late time action growth rate has the general form

$$
\text { two horizons: } \quad \begin{aligned}
\frac{d I}{d t}= & \left.\left(-(1-\gamma) \Phi_{e} Q_{e}-\gamma \Phi_{m} Q_{m}\right)\right|_{r_{-}} ^{r_{+}} ; \\
\text {one horizon: } \quad \frac{d I}{d t}= & 2 M-(1-\gamma) \Phi_{e+} Q_{e}-\gamma \Phi_{m+} Q_{m} \\
& +U\left(Q_{e}^{2}+Q_{m}^{2}\right)+(1-2 \gamma)\left(Q_{e}^{2}-Q_{m}^{2}\right) V\left(Q_{e}^{2}+Q_{m}^{2}\right),
\end{aligned}
$$

where $U$ and $V$ functions depend on the detail of a specific theory. These examples suggest that $\gamma=\frac{1}{2}$ is universal for restoring the electromagnetic duality in the holographic complexity from the CA conjecture. The result can be easily established for theories involving only the linear Maxwell equations, but an abstract proof is still lacking for general nonlinear field equations. However, our explicit demonstration that $\gamma=\frac{1}{2}$ holds for the EBI theory strongly suggests that it is indeed universal. It is of great interest to give a general proof or investigate further examples.

Our results do not address the question of which action equals the holographic complexity, raised in [44]. For theories with electromagnetic duality, we may require $\gamma=\frac{1}{2}$ to fix the action. However, even in this case, we can add more terms such as $F \wedge F$ into the action that respect the duality. For the RN-AdS black hole, the most general possibility of the holographic complexity based on CA that respects the electromagnetic duality is given by (3.16) with arbitrary coefficient $\tilde{\gamma}$. When the bulk theory itself does not have the electromagnetic duality, (e.g. including $\left(F^{2}\right)^{2}$ term, ) there is, a priori, no justification even to fix $\gamma=\frac{1}{2}$ and it is of great interest to investigate whether $\gamma=\frac{1}{2}$ is still special. Regardless the outcome, the CA conjecture requires some further refinements.

\section{Acknowledgments}

H.-S.L. is supported in part by NSFC (National Natural Science Foundation of China) Grants No. 11475148 and No. 11675144. H.L. is supported in part by NSFC Grants No. 11875200 and No. 11475024.

Open Access. This article is distributed under the terms of the Creative Commons Attribution License (CC-BY 4.0), which permits any use, distribution and reproduction in any medium, provided the original author(s) and source are credited. 


\section{References}

[1] J.M. Maldacena, The Large-N limit of superconformal field theories and supergravity, Int. J. Theor. Phys. 38 (1999) 1113 [Adv. Theor. Math. Phys. 2 (1998) 231] [hep-th/9711200] [INSPIRE].

[2] S.S. Gubser, I.R. Klebanov and A.M. Polyakov, Gauge theory correlators from noncritical string theory, Phys. Lett. B 428 (1998) 105 [hep-th/9802109] [INSPIRE].

[3] E. Witten, Anti-de Sitter space and holography, Adv. Theor. Math. Phys. 2 (1998) 253 [hep-th/9802150] [INSPIRE].

[4] O. Aharony, S.S. Gubser, J.M. Maldacena, H. Ooguri and Y. Oz, Large N field theories, string theory and gravity, Phys. Rept. 323 (2000) 183 [hep-th/9905111] [InSPIRE].

[5] S.A. Hartnoll, Lectures on holographic methods for condensed matter physics, Class. Quant. Grav. 26 (2009) 224002 [arXiv:0903.3246] [INSPIRE].

[6] S. Sachdev, What can gauge-gravity duality teach us about condensed matter physics?, Ann. Rev. Condensed Matter Phys. 3 (2012) 9 [arXiv:1108.1197] [InSPIRE].

[7] J. McGreevy, TASI 2015 Lectures on Quantum Matter (with a View Toward Holographic Duality), in proceedings of the Theoretical Advanced Study Institute in Elementary Particle Physics: New Frontiers in Fields and Strings (TASI 2015), Boulder, CO, U.S.A., 1-26 June 2015, pp. 215-296 [https://doi.org/10.1142/9789813149441_0004] [arXiv:1606.08953] [INSPIRE].

[8] J. Zaanen, Y.W. Sun, Y. Liu and K. Schalm, Holographic duality in condensed matter physics, Cambridge University Press, Cambridge U.K. (2015) [INSPIRE].

[9] L. Susskind, Computational Complexity and Black Hole Horizons, Fortsch. Phys. 64 (2016) 24 [Addendum ibid. 64 (2016) 44] [arXiv:1402.5674] [INSPIRE].

[10] D. Stanford and L. Susskind, Complexity and Shock Wave Geometries, Phys. Rev. D 90 (2014) 126007 [arXiv:1406.2678] [INSPIRE].

[11] A.R. Brown, D.A. Roberts, L. Susskind, B. Swingle and Y. Zhao, Holographic Complexity Equals Bulk Action?, Phys. Rev. Lett. 116 (2016) 191301 [arXiv:1509.07876] [INSPIRE].

[12] A.R. Brown, D.A. Roberts, L. Susskind, B. Swingle and Y. Zhao, Complexity, action and black holes, Phys. Rev. D 93 (2016) 086006 [arXiv: 1512.04993] [InSPIRE].

[13] L. Lehner, R.C. Myers, E. Poisson and R.D. Sorkin, Gravitational action with null boundaries, Phys. Rev. D 94 (2016) 084046 [arXiv: 1609.00207] [InSPIRE].

[14] D. Carmi, R.C. Myers and P. Rath, Comments on Holographic Complexity, JHEP 03 (2017) 118 [arXiv:1612.00433] [INSPIRE].

[15] A. Reynolds and S.F. Ross, Divergences in Holographic Complexity, Class. Quant. Grav. 34 (2017) 105004 [arXiv:1612.05439] [INSPIRE].

[16] Y. Zhao, Complexity and Boost Symmetry, Phys. Rev. D 98 (2018) 086011 [arXiv: 1702.03957] [INSPIRE].

[17] S.-J. Zhang, Complexity and phase transitions in a holographic QCD model, Nucl. Phys. B 929 (2018) 243 [arXiv: 1712.07583] [INSPIRE].

[18] J. Jiang and H.-B. Zhang, Surface term, corner term and action growth in $F\left(R_{a b c d}\right)$ gravity theory, Phys. Rev. D 99 (2019) 086005 [arXiv:1806.10312] [INSPIRE]. 
[19] Z.-Y. Fan and M. Guo, Holographic complexity under a global quantum quench, arXiv: 1811.01473 [INSPIRE].

[20] J. Jiang, Action growth rate for a higher curvature gravitational theory, Phys. Rev. D 98 (2018) 086018 [arXiv: 1810.00758] [INSPIRE].

[21] S.A. Hosseini Mansoori, V. Jahnke, M.M. Qaemmaqami and Y.D. Olivas, Holographic complexity of anisotropic black branes, arXiv:1808.00067 [INSPIRE].

[22] H. Ghaffarnejad, M. Farsam and E. Yaraie, Effects of quintessence dark energy on the action growth and butterfly velocity, arXiv:1806.05735 [INSPIRE].

[23] E. Yaraie, H. Ghaffarnejad and M. Farsam, Complexity growth and shock wave geometry in AdS-Maxwell-power-Yang-Mills theory, Eur. Phys. J. C 78 (2018) 967 [arXiv: 1806.07242] [INSPIRE].

[24] Y.-S. An and R.-H. Peng, Effect of the dilaton on holographic complexity growth, Phys. Rev. D 97 (2018) 066022 [arXiv:1801.03638] [INSPIRE].

[25] J. Jiang, Holographic complexity in charged Vaidya black hole, Eur. Phys. J. C 79 (2019) 130 [arXiv: 1811.07347] [INSPIRE].

[26] Seth Lloyd, Ultimate physical limits to computation, Nature 406 (2000) 1047 [quant-ph/9908043].

[27] R.-G. Cai, S.-M. Ruan, S.-J. Wang, R.-Q. Yang and R.-H. Peng, Action growth for AdS black holes, JHEP 09 (2016) 161 [arXiv: 1606.08307] [INSPIRE].

[28] H. Huang, X.-H. Feng and H. Lü, Holographic Complexity and Two Identities of Action Growth, Phys. Lett. B 769 (2017) 357 [arXiv:1611.02321] [INSPIRE].

[29] W.-J. Pan and Y.-C. Huang, Holographic complexity and action growth in massive gravities, Phys. Rev. D 95 (2017) 126013 [arXiv: 1612.03627] [INSPIRE].

[30] M. Alishahiha, A. Faraji Astaneh, A. Naseh and M.H. Vahidinia, On complexity for $F(R)$ and critical gravity, JHEP 05 (2017) 009 [arXiv:1702.06796] [INSPIRE].

[31] P. Wang, H. Yang and S. Ying, Action growth in $f(R)$ gravity, Phys. Rev. D 96 (2017) 046007 [arXiv: 1703.10006] [INSPIRE].

[32] W.-D. Guo, S.-W. Wei, Y.-Y. Li and Y.-X. Liu, Complexity growth rates for AdS black holes in massive gravity and $f(R)$ gravity, Eur. Phys. J. C 77 (2017) 904 [arXiv:1703.10468] [INSPIRE].

[33] P.A. Cano, R.A. Hennigar and H. Marrochio, Complexity Growth Rate in Lovelock Gravity, Phys. Rev. Lett. 121 (2018) 121602 [arXiv:1803.02795] [INSPIRE].

[34] R.-G. Cai, M. Sasaki and S.-J. Wang, Action growth of charged black holes with a single horizon, Phys. Rev. D 95 (2017) 124002 [arXiv:1702.06766] [INSPIRE].

[35] R.-Q. Yang, C. Niu, C.-Y. Zhang and K.-Y. Kim, Comparison of holographic and field theoretic complexities for time dependent thermofield double states, JHEP 02 (2018) 082 [arXiv: 1710.00600] [INSPIRE].

[36] X.-H. Feng and H.-S. Liu, Holographic Complexity Growth Rate in Horndeski Theory, Eur. Phys. J. C 79 (2019) 40 [arXiv:1811.03303] [INSPIRE].

[37] J. Couch, W. Fischler and P.H. Nguyen, Noether charge, black hole volume and complexity, JHEP 03 (2017) 119 [arXiv:1610.02038] [INSPIRE]. 
[38] Z.-Y. Fan and M. Guo, On the Noether charge and the gravity duals of quantum complexity, JHEP 08 (2018) 031 [arXiv: 1805.03796] [INSPIRE].

[39] J. Jiang and X.-W. Li, Modified "complexity equals action" conjecture, arXiv:1903.05476 [INSPIRE].

[40] D. Momeni, M. Faizal, S. Bahamonde and R. Myrzakulov, Holographic complexity for time-dependent backgrounds, Phys. Lett. B 762 (2016) 276 [arXiv:1610.01542] [inSPIRE].

[41] M. Alishahiha, Holographic Complexity, Phys. Rev. D 92 (2015) 126009 [arXiv:1509.06614] [INSPIRE].

[42] Z.-Y. Fan and M. Guo, Holographic complexity and thermodynamics of AdS black holes, Phys. Rev. D 100 (2019) 026016 [arXiv:1903.04127] [INSPIRE].

[43] A.R. Brown, H. Gharibyan, H.W. Lin, L. Susskind, L. Thorlacius and Y. Zhao, Complexity of Jackiw-Teitelboim gravity, Phys. Rev. D 99 (2019) 046016 [arXiv:1810.08741] [INSPIRE].

[44] K. Goto, H. Marrochio, R.C. Myers, L. Queimada and B. Yoshida, Holographic Complexity Equals Which Action?, JHEP 02 (2019) 160 [arXiv:1901.00014] [INSPIRE].

[45] S. Deser and C. Teitelboim, Duality Transformations of Abelian and Nonabelian Gauge Fields, Phys. Rev. D 13 (1976) 1592 [inSPIRE].

[46] S. Deser, Off-Shell Electromagnetic Duality Invariance, J. Phys. A 15 (1982) 1053 [INSPIRE].

[47] S. Deser, M. Henneaux and C. Teitelboim, Electric-magnetic black hole duality, Phys. Rev. D 55 (1997) 826 [hep-th/9607182] [INSPIRE].

[48] E. Cremmer, B. Julia, H. Lü and C.N. Pope, Dualization of dualities. 2. Twisted self-duality of doubled fields and superdualities, Nucl. Phys. B 535 (1998) 242 [hep-th/9806106] [INSPIRE].

[49] G.W. Gibbons and S.W. Hawking, Action Integrals and Partition Functions in Quantum Gravity, Phys. Rev. D 15 (1977) 2752 [inSPIRE].

[50] H.W. Braden, J.D. Brown, B.F. Whiting and J.W. York Jr., Charged black hole in a grand canonical ensemble, Phys. Rev. D 42 (1990) 3376 [INSPIRE].

[51] S.W. Hawking and S.F. Ross, Duality between electric and magnetic black holes, Phys. Rev. D 52 (1995) 5865 [hep-th/9504019] [INSPIRE].

[52] H. Lü, Y. Pang and C.N. Pope, AdS Dyonic Black Hole and its Thermodynamics, JHEP 11 (2013) 033 [arXiv:1307.6243] [INSPIRE].

[53] C.J. Gao and S.N. Zhang, Dilaton black holes in de Sitter or Anti-de Sitter universe, Phys. Rev. D 70 (2004) 124019 [hep-th/0411104] [INSPIRE].

[54] H. Lü, Charged dilatonic AdS black holes and magnetic $A d S_{D-2} \times R^{2}$ vacua, JHEP 09 (2013) 112 [arXiv: 1306. 2386] [INSPIRE].

[55] M. Born and L. Infeld, Foundations of the new field theory, Proc. Roy. Soc. Lond. A 144 (1934) 425 [INSPIRE].

[56] A. García D, H. Salazar I and J.F. Plebański, Type-D solutions of the Einstein and Born-Infeld nonlinear electrodynamics equations, Nuovo Cim. B 84 (1984) 65.

[57] S. Li, H. Lü and H. Wei, Dyonic (A)dS Black Holes in Einstein-Born-Infeld Theory in Diverse Dimensions, JHEP 07 (2016) 004 [arXiv: 1606.02733] [INSPIRE].

[58] G.W. Horndeski, Second-order scalar-tensor field equations in a four-dimensional space, Int. J. Theor. Phys. 10 (1974) 363 [INSPIRE]. 
[59] D. Lovelock, The Einstein tensor and its generalizations, J. Math. Phys. 12 (1971) 498 [INSPIRE].

[60] A. Anabalon, A. Cisterna and J. Oliva, Asymptotically locally AdS and flat black holes in Horndeski theory, Phys. Rev. D 89 (2014) 084050 [arXiv:1312.3597] [InSPIRE].

[61] A. Cisterna and C. Erices, Asymptotically locally AdS and flat black holes in the presence of an electric field in the Horndeski scenario, Phys. Rev. D 89 (2014) 084038 [arXiv: 1401.4479] [INSPIRE].

[62] X.-H. Feng, H.-S. Liu, H. Lü and C.N. Pope, Black Hole Entropy and Viscosity Bound in Horndeski Gravity, JHEP 11 (2015) 176 [arXiv:1509.07142] [INSPIRE].

[63] X.-H. Feng, H.-S. Liu, H. Lü and C.N. Pope, Thermodynamics of Charged Black Holes in Einstein-Horndeski-Maxwell Theory, Phys. Rev. D 93 (2016) 044030 [arXiv:1512.02659] [INSPIRE].

[64] J. Beltran Jimenez, R. Durrer, L. Heisenberg and M. Thorsrud, Stability of Horndeski vector-tensor interactions, JCAP 10 (2013) 064 [arXiv:1308.1867] [INSPIRE].

[65] T. Kobayashi, H. Motohashi and T. Suyama, Black hole perturbation in the most general scalar-tensor theory with second-order field equations II: the even-parity sector, Phys. Rev. D 89 (2014) 084042 [arXiv:1402.6740] [INSPIRE].

[66] M. Minamitsuji, Causal structure in the scalar-tensor theory with field derivative coupling to the Einstein tensor, Phys. Lett. B 743 (2015) 272 [INSPIRE].

[67] X.-M. Kuang and E. Papantonopoulos, Building a Holographic Superconductor with a Scalar Field Coupled Kinematically to Einstein Tensor, JHEP 08 (2016) 161 [arXiv:1607.04928] [INSPIRE].

[68] W.-J. Jiang, H.-S. Liu, H. Lü and C.N. Pope, DC Conductivities with Momentum Dissipation in Horndeski Theories, JHEP 07 (2017) 084 [arXiv: 1703.00922] [INSPIRE].

[69] M. Baggioli and W.-J. Li, Diffusivities bounds and chaos in holographic Horndeski theories, JHEP 07 (2017) 055 [arXiv: 1705.01766] [INSPIRE].

[70] H.-S. Liu, H. Lü and C.N. Pope, Holographic Heat Current as Noether Current, JHEP 09 (2017) 146 [arXiv : 1708.02329] [INSPIRE].

[71] X.-H. Feng, H.-S. Liu, W.-T. Lu and H. Lü, Horndeski Gravity and the Violation of Reverse Isoperimetric Inequality, Eur. Phys. J. C 77 (2017) 790 [arXiv:1705.08970] [INSPIRE].

[72] E. Caceres, R. Mohan and P.H. Nguyen, On holographic entanglement entropy of Horndeski black holes, JHEP 10 (2017) 145 [arXiv:1707.06322] [INSPIRE].

[73] W.-J. Geng, S.-L. Li, H. Lü and H. Wei, Gödel metrics with chronology protection in Horndeski gravities, Phys. Lett. B $\mathbf{7 8 0}$ (2018) 196 [arXiv:1801.00009] [INSPIRE].

[74] Y.-Z. Li and H. Lü, a-theorem for Horndeski gravity at the critical point, Phys. Rev. D 97 (2018) 126008 [arXiv: 1803.08088] [INSPIRE].

[75] H.-S. Liu, Violation of Thermal Conductivity Bound in Horndeski Theory, Phys. Rev. D 98 (2018) 061902 [arXiv:1804.06502] [INSPIRE].

[76] Y.-Z. Li, H. Lü and H.-Y. Zhang, Scale Invariance vs. Conformal Invariance: Holographic Two-Point Functions in Horndeski Gravity, Eur. Phys. J. C 79 (2019) 592 [arXiv: 1812.05123] [INSPIRE]. 